\title{
Graph-analytical interpretation and modeling of phase portraits of cellular structures during their phase transformations
}

\author{
Vladimir Kulikov ${ }^{1, *}$ \\ ${ }^{1}$ Moscow State University of Civil Engineering; 26, Yaroslavskoe Shosse, Moscow, 129337, Russia
}

\begin{abstract}
The article shows the possibility of representing the state of the cellular structures in the form of "phase portraits" of states in discrete moments of time. The generated structures are presented in the form of dynamic systems. Models of states of dynamical systems, is developed, based on the analysis and processing of laser speckle and other structures. The simulation algorithm of the phase portraits given in the paper allows to create information systems casts at given discrete points in time, and to control the process of formation of properties of structures. The transition of the studied systems from their initial states to the final presents a recursive algorithm with deferred calculation of the parameters of designed objects. The initial parameters of the studied systems are reported in the form of a graphic-analytical statistics and are defined further as texture geometric shapes of cellular structures.
\end{abstract}

\section{Introduction}

Cell structure studied by the author, are a dynamic system. The term "dynamic system" we mean any object or process that clearly defined the concept of a state as a set of some values in the initial time, and set a law describing the evolution of the initial state over time. The structure of the term is schematically shown in Fig.1.

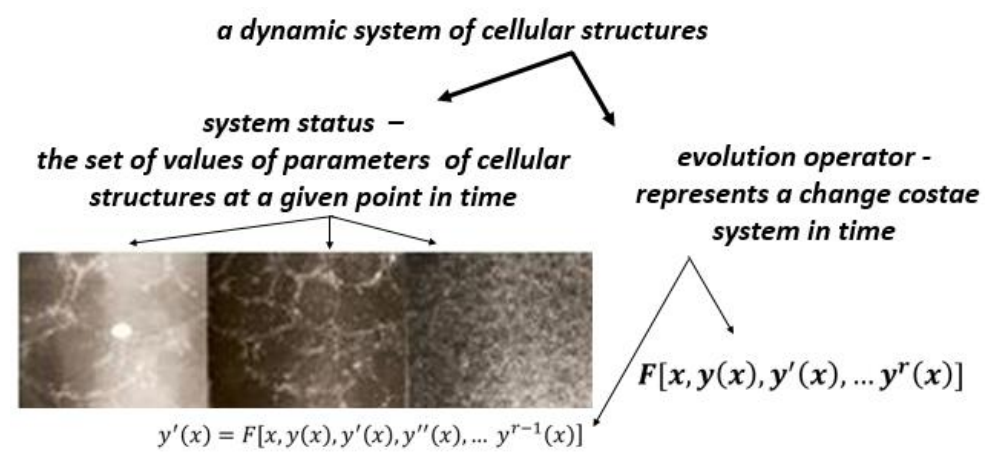

Fig.1. The structure of the notion "dynamic system" of cellular structure.

\footnotetext{
${ }^{*}$ Corresponding author: kulikov-miit@mail.ru
} 
The evolution of the system is shown in Fig.1 can be formalized by a nonlinear operator which connects generalized forces and generalized coordinates. If $\mathrm{M}$ - region of Euclidean space (space whose properties are described by axioms of Euclidean geometry), and ( $\mathrm{t}$ ) is a continuously varying parameter, - evolution can be given by a system of ordinary differential equations (ODE). Changing the state of a system can be described by a system of ODE:

$$
x_{1}^{\prime}=f_{1}\left(x_{1}, \ldots, x_{n}\right), \ldots, x_{1}^{\prime}=f_{1}\left(x_{1}, \ldots, x_{n}\right)
$$

Variables $\mathrm{x}_{1}, \mathrm{x}_{2}, \ldots, \mathrm{x}_{\mathrm{n}}$ determine the state of the system at a particular instant of time, Fig. 2.

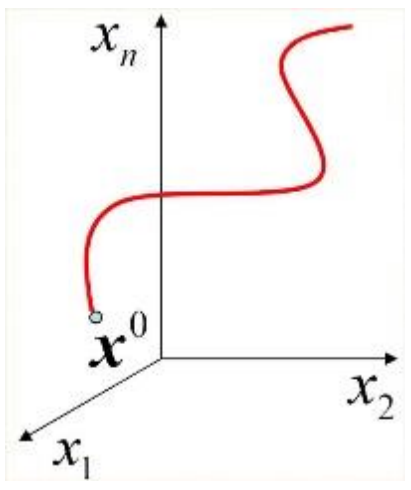

Fig. 2. State, representation of a cellular structure in Euclidean space.

A mathematical model of the dynamic system cellular structure will assume a form of writing, which: 1) - input parameters (coordinates) describing the system state and 2) given the operator $\left(\phi_{t}\right)$, allowing you to set the change of coordinates in time:

$$
F\left[x, y(x), y^{\prime}(x), \ldots y^{r}(x)\right] \rightarrow y^{\prime}(x)=F\left[x, y(x), y^{\prime}(x), y^{\prime \prime}(x), \ldots y^{r-1}(x)\right]
$$

The decision (1.2) will be presented in the form of the Cauchy problem which consists in finding a solution that meets the $r$ initial conditions. The formulation of the Cauchy problem for multiple parameters of cellular structures, are defined as follows:

$$
\frac{d y_{1}}{d t}=\left(a-b \cdot y_{2}\right) \cdot y_{1} ; \quad \frac{d y_{1}}{d t}=\left(-c+d \cdot y_{1}\right) \cdot y_{2} ; \quad y_{1}=3 ; \quad y_{2}=1
$$

Cellular structures represent artificial stone materials, which are composed of a binder, $(C)$, as well as different shapes and sizes of cells, Fig. 3. As the first binder can be coupled to a filler. Cells thus are distributed throughout the volume of artificial stone is relatively uniform. Many types of cellular materials differ in method of formation of porous structure, and the type of binder. The difference is in the method of hardening, and also in terms of the molding, i.e. the production technology (PT). Physico-mechanical characteristics (P$\mathrm{MF}$ ) of the material also depend on the technologies used in its production. Thus obtained materials with various size cells. The cells can be closed, communicating and open.

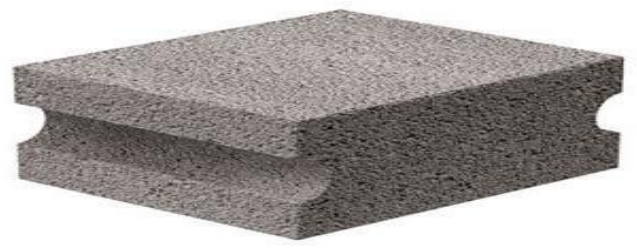

Fig. 3. The product a cellular structure. 
In the considered cellular structures, the role of small (FA) or coarse aggregate $(C A)$ can play the air bubbles. Previously they were referred to as cells. In some cases, the composition of the material introduced large-fractional filler. They may perlite, slag pumice, expanded clay, vermiculite or other expanded materials. Such components are referred to as wire mesh and light $(C L C)$.

Artificial stones can be classified according to the method of hardening. On this basis, produce the structure of the artificial $(A H)$ and natural hardening $(N H)$. The latter option is gaining strength under normal atmospheric conditions. Faux exposed to high temperature and water vapor. Autoclave method assumes a vapour pressure greater than 1 ATM., and temperature more than $100^{\circ} \mathrm{C}$. Another method involves a pressure less than 1 ATM. and temperature in the range of $25-100^{\circ} \mathrm{C}$.

A number of properties of cellular structures obey generalized laws. For example, the coefficient of thermal conductivity $(\lambda)$ depends on the volume weight $(Y)$ of the final product, but it is not affected by the kind of binder, and conditions of hardening. This is because the material of the walls that form the pores, is composed of calcium silicate or similar characteristics of cement stone. However, the porosity $(P)$ and volume weight $(Y)$ significantly affects the conductivity.

The strength of cellular structure (S) depends on the binder component, and the hardening condition. The best strength values has an autoclave structure, their strength is more in comparison with that which have natural hardening, the difference can differ by 10 times. The strength of the walls of the pores is determined by the amount of water used when mixing the initial mixture with water. Hardening of the structure, which was made on the basis of Portland cement, only some of the liquid will be involved in the process. The volume of water (W) during hydration of cement will depend on the mineralogical composition and will be equal to $15-20 \%$ of the weight of cement. The excess amount of water acts on the cement particles, promotes the formation of layers in the stone. After drying and water consumption on the hydration processes in the body structures are voids, cavities and pores, as well as air channels. A number of them appears in the result of drying the gel-like masses that arise in the process of hardening of cement. For this reason, cellular structure, standard (GOST) which was observed in their manufacture, can lose their strength with increasing volume of water.

\section{Materials and methods}

All of the above clearly demonstrates the complex structure of the cellular structures, a large variety of factors affecting their performance characteristics and of the difficulties of formalization. However, it can be assumed that the consumer quality (CQ) cellular structures are a function of the properties and quantities of the starting components, and also technologies of their forming and fabrication. All this can be presented as an unordered set (veriaty) (CQ):

$$
C Q=\{C, P T, P-M F, F A, C A, A H, N H, P, \lambda, \Upsilon, S, W, \ldots\}
$$

How it would be possible to preserve and pass on in time the positive properties of the original components and their combinations and properties in cellular structures formed in the process of their production technology? The author proposes to implement it with the help of laser and other speckle-structures (phase portraits), i.e. the information models of the studied systems at specific points in time. The texture of these information models in these discrete moments of time, to be a priori must conform to their inherent properties. Analytical dependences between the different elements of the set (2.1) can be obtained as a result of numerical calculation of the speckle patterns, presenting the speckle structure temporary visual side of their temporary condition as a temporary state of a dynamic 
system with inherent specific properties at specific points in time. This will allow you to control the process of formation of cellular structures by address (exact) changes of initial and current parameters of the initial components on the basis of obtained analytical dependencies.

Thus, using visual assessments of the above systems (No. 1 ... No. 8 Fig.4.) you can control the formation of the properties of cellular structures, through the creation of information models in the form of phase portraits of the system at specific points in time for different combinations of the elements of the set (2.1). In addition, using phase portraits it is possible to evaluate the impact of combinations of properties (2.1) on the consumer properties of cellular structures.

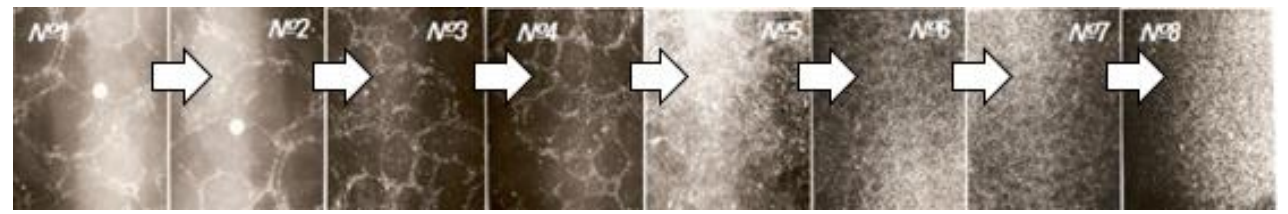

Fig. 4. Investigated the speckle structure at discrete points in time.

Reveal the concept of "laser speckle structure". When the observer examines in laser light diffusely reflecting or transmissive object, the image seems grainy to him. The impression that the object surface is covered with numerous small, randomly distributed bright and dark spots called speckles. If the observer focus his eyes or optical instrument to a point located before the object or behind it, the speckle structure remains visible. If the observer moves, then it seems that speckle-pattern flickers and shifts relative to the object. The phenomenon of speckles associated with the use of highly coherent light. As one of the major advantages of holographic interferometry is that it provides the opportunity to study diffuse objects, it is useful to know some basic properties of laser speckle-structures. Consider the concept of "lightness", "contrast" and "characteristic size" of the speckles.

In Fig.5. presents the speckles produced by scattering of the reflected laser light from a rough surface.

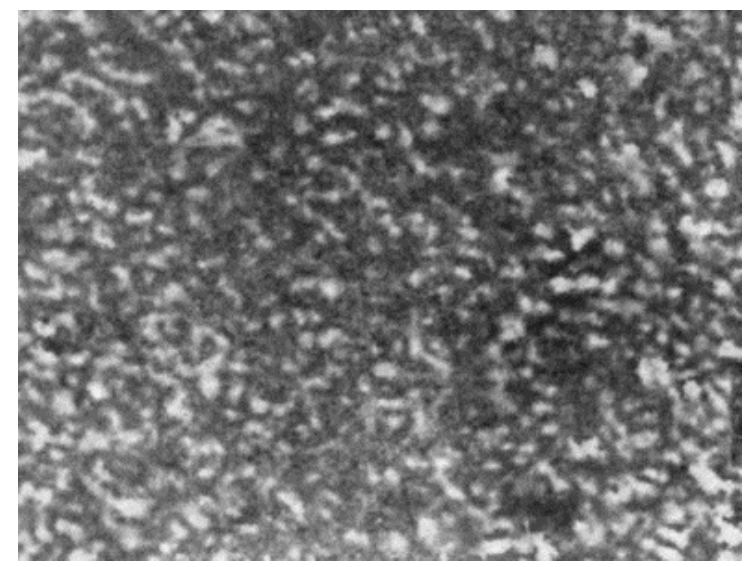

Fig. 5. The typical picture of laser speckles.

Under a rough surface we mean a surface having microscopic random variation of the terrain, the scale of which exceeds the wavelength of light. Almost all surfaces are rough. Speckles occur when light passes through the scattering surface, including using liquid. It is known that the honeycomb structure as a dynamic system sequentially pass through the different phases is a liquid phase state, becoming the ultimate in artificial stone - i.e. a solid phase. 
The physical nature of the speckles is simple. Each point of the object scatters some light towards the observer. Owing to its high coherence of laser light, diffused one of the points of the object interferes with the light scattered at any other point of the object. A detector such as a photo-film or the retina of the human eye, as the observer is placed in the light field, can register a picture of a chaotic interference pattern. This is speckles. The randomness due to the surface roughness, since the phase of the scattered light varies randomly from point to point, following the height variations of the terrain in this place.

To describe a speckle pattern quantitatively, we will consider her education in the scattering of laser light by the surface microrelief which is shown in Fig.6. We calculate statistical characteristics of illumination in the image plane.

\section{Results}

Instead of considering changes in luminance from point to point in this plane, it is convenient to consider ensemble average for a single point on the surface of the detector. In other words, we assume that the detector remains stationary in the position $\{x, y, z)$, and will measure the illumination at such points from each set (ensemble) of diffusers, which are macroscopically identical to each other, but microscopic are different from each other. Surfaces of all diffusers have the same statistical characteristics, e.g. the same mean-square surface elevation. We assume that the incident light is monochromatic and linearly polarized, and that polarization in the scattering is not changed. Imagine the complex amplitude of light reaching the detector as:

$$
U(x, y, z)=a(x, y, z) \cdot e^{-i \cdot \varphi(x, y, z)}
$$

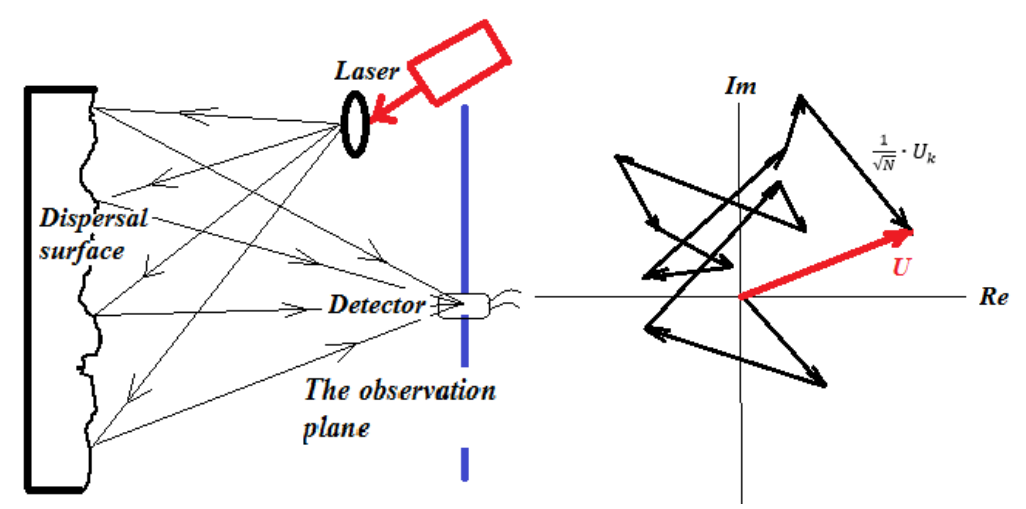

Fig. 6. Diagram of laser micro-relief.

Fig.7. The addition of random complex amplitudes.

And present it as a vector in the complex plane, as shown in Fig.7. This complex amplitude actually is the sum of a large number of $U$ components corresponding to the light incoming to the detector from the neighborhood of each point of a diffuse surface. Denoting the k-th component through $[\mathrm{N}-(1 / 2) \mathrm{U}(\mathrm{x}, \mathrm{y}, \mathrm{z})]$, we get:

$$
U(x, y, z)=\frac{\sum_{k=1}^{N} U(x, y, z)}{\sqrt{N}}=\frac{1}{\sqrt{N}} \cdot \sum_{k=1}^{N} a(x, y, z) \cdot e^{-i \cdot \varphi(x, y, z)}
$$

Calculate basic statistical properties of the light field speckle-structure. This analysis is essentially equivalent to the classical problem of two-dimensional random walk. In our case the random walk is performed in the complex plane, Fig.7. It turns out that in this case the complex amplitude obeys the statistical law of Gauss. In particular, the density function of the joint probability of the real and imaginary parts of the function $U$ has the form: 


$$
P_{r, i}\left(U^{r}, U^{i}\right)=\frac{1}{2 \cdot \pi \cdot \sigma^{2}} \cdot e^{\left[-\frac{\left(U^{r}\right)+\left(U^{i}\right)}{2 \cdot \sigma^{2}}\right]}
$$

Where

$$
\sigma=\lim _{N \rightarrow \infty}\left(\frac{1}{N}\right) \sum_{k=1}^{N} \frac{1}{2}\left\langle\left|U_{k}\right|^{2}\right\rangle
$$

We are most interested in the illumination intensity $\mathrm{I}=\mathrm{UU}^{*}$, because that is the value measured in practice. As is known, the expression (3.3) can be transformed to obtain the probability density function for light:

$$
P(I)=\frac{1}{\langle I\rangle} \cdot e^{\left(-\frac{1}{\langle I\rangle}\right)}
$$

Thus, the illuminance distribution in the image speckles obeys the statistical law with a negative exponent of the exponential. The most probable value of the illumination speckle is zero, which indicates a black speckle.

The amount of contrast of speckle structure will serve ratio $C=\frac{\sigma_{I}}{\langle I\rangle}$ where $\sigma \mathrm{I}$ is the standard deviation of the illuminance from the mean. For the distribution described by expression (3.5), the contrast of the speckle structure is equal to one.

To study cellular structures and visual presentation, ongoing dynamic processes use the concept of "phase space" in which we will construct the phase portraits of the structural conditions of the systems depicted in Fig.4. Each of the shown in Fig.4. dynamic systems has its own phase portrait. Analyze a speckle pattern No. 1 of Fig.8.

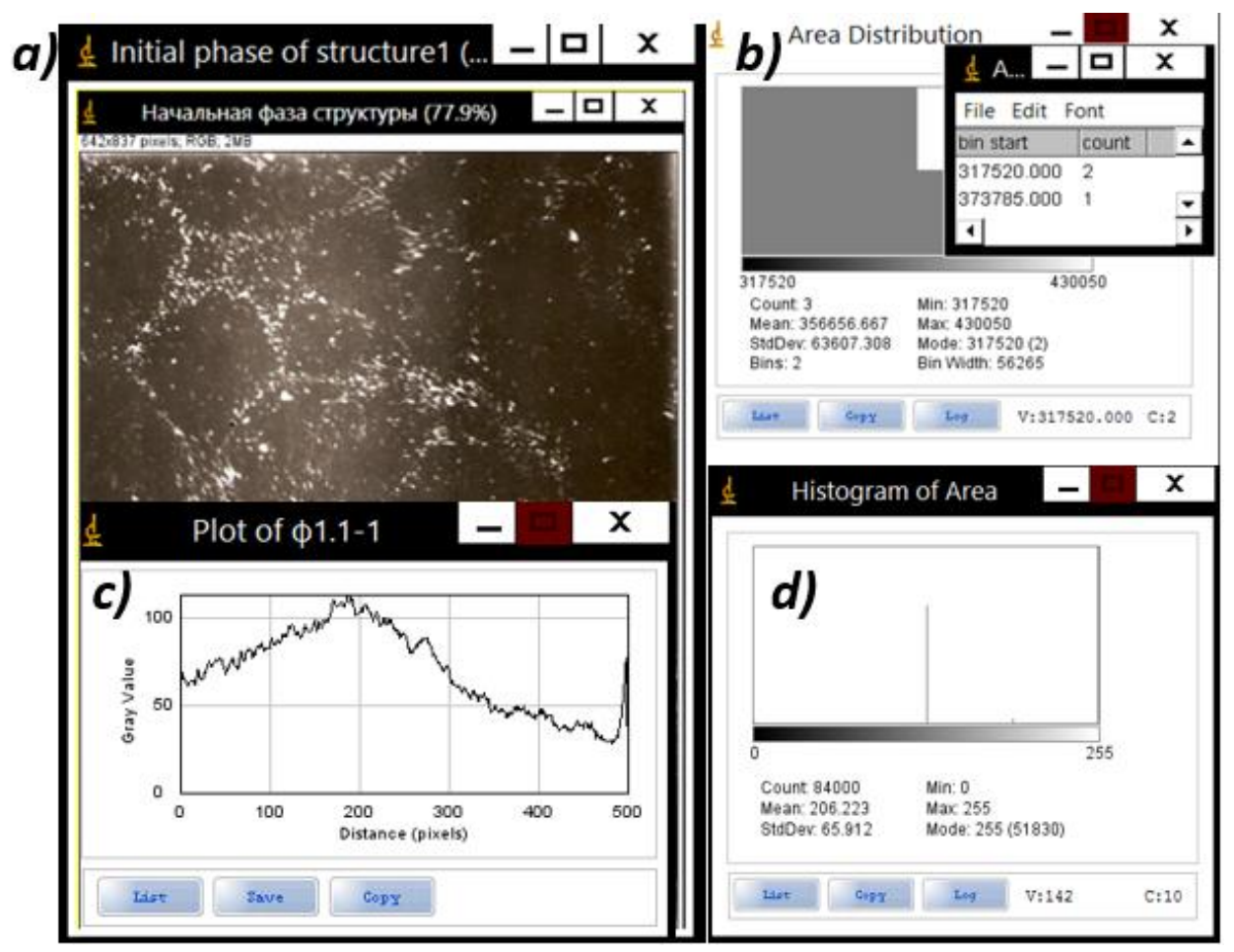

Fig. 8. Statistical processing of the speckle structure No. 1.

In Fig.8.and shows a speckle structure No. 1 cellular system of the set (2.1) at a given particular time. In the above illustration clearly observed the so-called singular points, "saddle", "center", "separates" (Saddle — rough type of a singular point. ... Center — stable 
type 2. All the trajectories near the singular point closed. ... Often separates parts of the region of attraction of various attractors), i.e. so-called point of equilibrium, which helps to predict the behavior of a dynamic system in time. Basic statistical characteristics of the speckle structure has been calculated and is shown in Fig.8.b. In Fig.8s constructed horizontal profile of the modeled surface in the coordinates "Distance-Brightness" of the speckles in Fig.8d shows a histogram of the distribution of speckles the surface for their size.

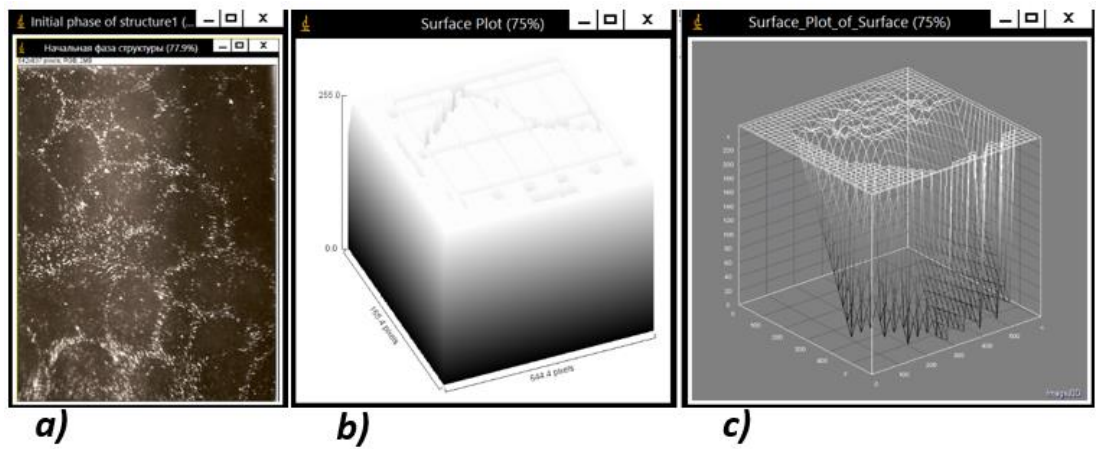

Fig. 9. Modeling scheme of the volumetric cellular structure on the basis of the speckle structure No. 1 .

a) shows the initial state of the speckle structure No. 1. b) shows the spatial substrate with a horizontal profile for a simulated surface; and c) presented finite-grid model based on the calculated statistical distribution data of the elements of the set (2.1)

Process flow consistent modeling volumetric cellular structure shown in Fig.9. The simulation algorithm is the following: - initially constructed horizontal profile of the speckle structure No. 1, which is then placed on the respective bulk substrate, which in the subsequent preobrazovaniya in a preliminary model of the speckle structure No. 1.

Further, in Fig. 10 (a) shows the preliminary model of the speckle structure No. 1, which is the basis of the phase portrait for the simulation of subsequent volumetric cellular structures. Thus, in Fig.10.b preliminary three-dimensional model of the speckle structure No. 1 presented with appropriate contour lines and their subsequent optimization, Fig.10c.

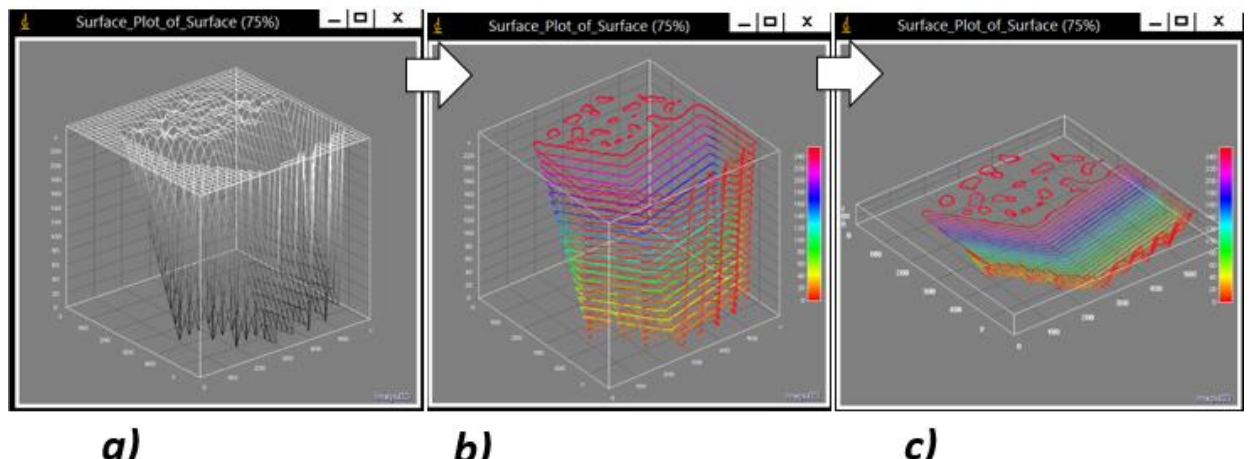

Fig. 10. The volumetric representation of the structures contour.

Note that the observed in Fig.9,10 singular points can be considered as a special equilibrium point, which in turn, can be stable or unstable. So, if the dynamic system is in the neighborhood of stable equilibrium points, small perturbations do not violate the stable operation of the system. If the point of equilibrium is not stable, the disturbance will progress which can lead to the destruction of the system. 


\section{Discussions}

This position will use for the forecast behavior of the investigated speckle-structure No. 1 .

We apply the method of phase space with respect to a dynamic system of second order of the set (2.1):

$$
\frac{d x}{d t}=F_{1}(x, y) ; \frac{d y}{d t}=F_{2}(x, y)
$$

here $\mathrm{F} 1(\mathrm{x}, \mathrm{y})$ and $\mathrm{F} 2(\mathrm{x}, \mathrm{y})$ function of its arguments, the sets (2.4).

To obtain the phase trajectories eliminate time from $(4.1,4.2)$. Divide the second equation (4.2) on the first (4.1)

$$
\frac{d y}{d x}=\frac{F_{2}(x, y)}{F_{1}(x, y)}
$$

At the point of equilibrium the time derivatives become zero and then we get the ratio of uncertainties:

$$
\frac{d y}{d x}=\frac{F_{2}(x, y)}{F_{1}(x, y)}=\frac{0}{0}
$$

The point at which there are uncertainties, are called singular points. At specific points in the phase plane of solutions can bifurcate that actually observed in Fig.8a.

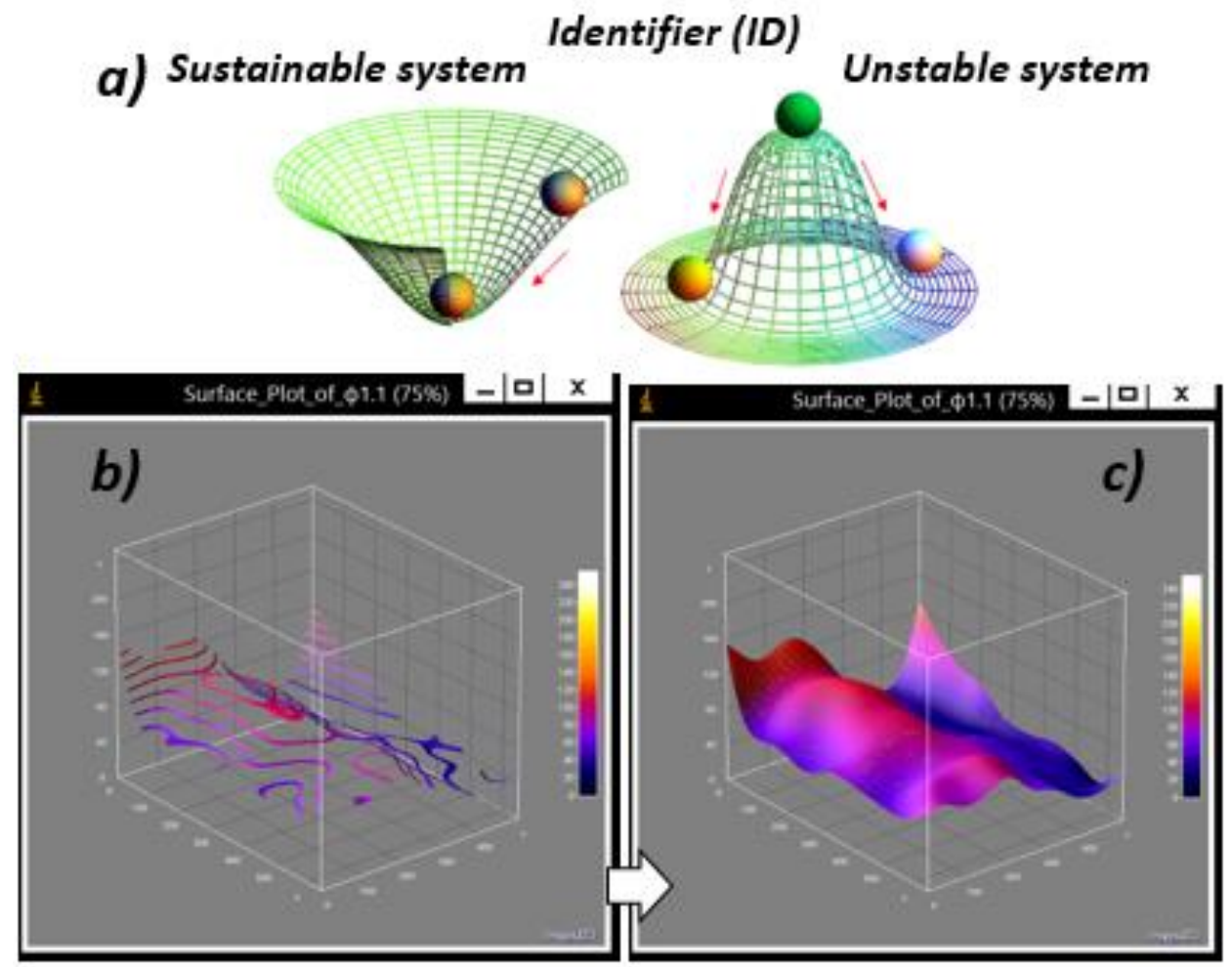

Fig. 11. Visual identification of the status of the speckle structure No. 1.

In Fig.11(a) shows a visual identifier (ID) indicating the probability of state a component of the set (2.1).

In Fig.11(b) shows the calculated and the characteristics of the contour of the studied structure No. 1, which is a picture of their projection on a horizontal surface, which in fact 
represents the phase portrait of the investigated structure No. 1 at this point in time. Based on these data, the developed information model is shown in Fig.11(c).

Comparing the information model in Fig.11(C) ID Fig.11(a) are the following:

1. The speckles in the coordinates on the X-axis from 0 to 270 and $\mathrm{Y}$ from 0 to 500 , visually fit the image of an unstable system.

2. The speckles in the coordinates on the $\mathrm{X}$-axis from 400 to 500 and $\mathrm{Y}$ is from 0 to 500 is also visually consistent with the concept of a stable system.

3. Thus, it is this interpretation of the speckle structure No. 1 is consistent with the information content of the image, because the study of the speckle structure corresponds to the situation at the beginning of the formation of the cellular structure in which the liquid phase of the system is unstable, gradually transformed into a specific pattern of calcium hydrosilicates (singular points), i.e. in a stable system.

\section{Conclusion}

Thus, in managed speckle-structure state No. 1, with its statistical parameters, the resulting graph-analytical analysis of dynamic systems is given in Fig.4., is to put into correspondence with the information model shown in Fig.11C as the phase portrait of system No. 1 at the specified time. Similarly, all shown in Fig.4 States cellular structure No. 1 ... No. 8 in the same way can be assigned to phase portraits that correspond to specific properties of the set (2.1).

\section{References}

1. J.L. Fiadeiro, A. Lopes, Theoretical Computer Science 663, 1-33 (2017)

2. F. Nian, W. Liu, Chaos, Solitons \& Fractals 91, 554-561 (2016)

3. Y.Wang, G. Chen, Optik - International Journal for Light and Electron Optics 132, 111-120 (2017)

4. G. Caruso, D. Vitale Di, Maio International Journal of Heat and Mass Transfer 68, 401-414 (2014)

5. Q. Zhang, X.Duan, Sh. Ma, Statistics \& Probability Letters 122, 11-19 (2017) 